\title{
Mining and evolution analysis of lateral organ boundaries domain (LBD) genes in Chinese white pear (Pyrus bretschneideri)
}

\author{
Bobo Song ${ }^{1 \dagger}$, Zikai Tang ${ }^{1 \dagger}$, Xiaolong $\mathrm{Li}^{2}$, Jiaming Li ${ }^{1}$, Mingyue Zhang ${ }^{1}$, Kejiao Zhao ${ }^{1}$, Hainan Liu', \\ Shaoling Zhang ${ }^{1}$ and Jun $\mathrm{Wu}^{1 *}$
}

\begin{abstract}
Background: The lateral organ boundaries domain $(\angle B D)$ gene is a plant-specific transcription factor that plays a critical role in diverse biological processes. However, the evolution and functional divergence of the $\angle B D$ gene family has not yet been characterized for the Chinese White Pear.

Results: In our study, a total of 60 PbrLBDs were identified in the pear genome. The PbrLBD gene family was divided into two classes based on gene structure and phylogenetic analysis: class I (53) and class II (7). Cis-acting element analysis results suggested that PbrLBDs may participate in various biological processes, such as flavonoid biosynthetic and stress response. Synteny analysis results indicated that segmental duplication played a key role in the expansion of the PbrLBD gene family. The mean Ks and 4DTv values showed that the PbrLBD gene family had undergone only one recent whole-genome duplication event occurring at 30-45 MYA. Purifying selection was a primary force during the PbrLBD gene family evolution process. Transcriptome data analysis revealed that 10 PbrLBDs were expressed in all six examined tissues, and $73.33 \%$ of members in the PbrLBD gene family were expressed in pear sepal. qRT-PCR was conducted to verify the expression levels of 11 PbrLBDs in these six tissues. Specifically, PbrLBD20, PbrLBD35 and PbrLBD53 genes were down-regulated when anthocyanin concentrations were high, whereas PbrLBD33 was significantly up-regulated in pear when anthocyanin concentrations were high. Furthermore, PbrLBD20, one of the candidate genes related to anthocyanins was localized in the nucleus.

Conclusions: Our analysis provides valuable information for understanding the evolution of the PbrLBD gene family, and provides new insights into the regulation of pear pigment metabolism and lays a foundation for the future disclosure of the molecular mechanism of $\angle B D$ gene regulating flavonoid metabolism.
\end{abstract}

Keywords: LBD gene family, Pyrus, Synteny analysis, Gene expression, Anthocyanins biosynthesis

\section{Background}

Lateral organ boundaries domain $(L B D)$ genes are plantspecific transcription factors (TFs) that play important roles in the growth and development of plants. They contribute to abiotic stress responses, anthocyanin biosynthesis,

\footnotetext{
* Correspondence: wujun@njau.edu.cn

${ }^{\dagger}$ Bobo Song and Zikai Tang contributed equally to this work.

'Center of Pear Engineering Technology Research, State Key Laboratory of Crop Genetics and Germplasm Enhancement, Nanjing Agricultural University, Nanjing 210095, China

Full list of author information is available at the end of the article
}

nitrogen metabolism and development of lateral organ, among other processes [1-4]. According to the structural characteristics of LBD, the $L B D$ genes were divided into two subclasses, class I and class II $[5,6]$. The class I genes encode a conserved LOB domain composed of a conserved CX2CX6CX3C zinc finger-like domain at the $\mathrm{N}$ terminal and a conserved LX6LX3LX6L leucine zipper-like domain $[5,7]$. However, the class II genes only contain a conserved CX2CX6CX3C zinc finger-like coiled-coil domain [8]. A previous study showed that the two class I domains are

(c) The Author(s). 2020 Open Access This article is licensed under a Creative Commons Attribution 4.0 International License, which permits use, sharing, adaptation, distribution and reproduction in any medium or format, as long as you give appropriate credit to the original author(s) and the source, provide a link to the Creative Commons licence, and indicate if changes were made. The images or other third party material in this article are included in the article's Creative Commons licence, unless indicated otherwise in a credit line to the material. If material is not included in the article's Creative Commons licence and your intended use is not permitted by statutory regulation or exceeds the permitted use, you will need to obtain permission directly from the copyright holder. To view a copy of this licence, visit http://creativecommons.org/licenses/by/4.0/ The Creative Commons Public Domain Dedication waiver (http://creativecommons.org/publicdomain/zero/1.0/) applies to the data made available in this article, unless otherwise stated in a credit line to the data. 
involved in different roles. The CX2CX6CX3C zinc fingerlike domain was associated with DNA binding, while the LX6LX3LX6L leucine zipper-like domain was used for protein-protein interactions [5].

The function of many $L B D$ genes has been previously identified in several model species. For example, AS2, which is related to the formation of flat symmetric leaves, is one of the most extensively investigated members of the $L B D$ gene family. In Arabidopsis thaliana, it was shown that Knotted1-like homeobox (KNOX) genes function in maintaining an active meristem. However, the AS1-AS2 complex was shown to promote meristem cell differentiation by repressing expression of KNOX genes. One previous study showed that ARF3 (ETTIN/ AUXIN RESPONSE FACTOR3) was an important determinant for specification of adaxial cells in leaves, while the expression of $A R F 3$ was directly repressed by $A S 2$ and AS1 [9]. This result indicated that $A S 2$ and $A S 1$ indirectly control the formation of adaxial cells in leaves. In addition, the development of plant roots also was regulated by several $L B D$ genes. As a member of the maize $L B D$ gene family, $r a 2$ can directly regulate the development of stem cells in maize branch meristems. The ramosa2 (ra2) mutant causes an increase in the number of branches and long branches to replace short branches compared with normal maize [10]. It has been previously shown that AtARF7 and AtARF19, as early auxin response factors, regulate the formation of the lateral root in Arabidopsis [3]. Recent studies further revealed that AtARF7 and AtARF19 directly regulate the expression of AtLBD16 and AtLBD29, which induce formation of the lateral root. Moreover, $A t L B D 18$ is also regulated by AtARF7 and AtARF19, and functions in lateral root formation. In rice, $A R L 1$ encodes a predicted protein containing a LOB domain with a broad range of expression in various tissues, including lateral and adventitious root primordia, tiller primordia, vascular tissues, scutellum and young pedicels. It also plays an important role in the development of adventitious roots. Meanwhile, several $L B D$ genes also are involved in nitrate metabolism and anthocyanin synthesis. For example, one study showed that ASL39/LBD37, ASL40/LBD38 and ASL41/ $L B D 39$ were induced by $\mathrm{N} / \mathrm{NO}_{3}{ }^{-}$, and negatively regulated anthocyanin biosynthesis in Arabidopsis thaliana [2].

To date, the $L B D$ gene family has been systematically investigated at the whole-genome level for some plants, such as Arabidopsis thaliana, maize (Zea mays), rice (Oryza sativa), poplar (Populus trichocarpa), apple (Malus domestica), grape (Vitis vinifera) and tomato (Solanum lycopersicum) [5, 11-14]. Pear, one of the most important Rosaceae fruit trees, is widely cultivated all over the world. However, till now there has been insufficient information on $L B D$ genes in pear, making functional characterization of $\operatorname{PbrLBD}$ genes difficult. The release of the Chinese White Pear genome (Pyrus bretschneideri) has provided an unprecedented opportunity to identify its $L B D$ gene family at the wholegenome level. In the present study, we performed comprehensive analysis on genome-wide identification, chromosome distribution, genomic structure, and evolutionary and expression patterns of $\operatorname{PbrLBD}$ genes, which will establish a solid foundation for functional characterization of PbrLBD genes in the future.

\section{Results \\ Whole-genome characterization of $P b r L B D$ genes in Chinese white pear}

In the present study, two strategies were independently performed to identify the candidate $L B D$ gene set in the pear genome. First, a Hidden Markov Model search (HMMsearch) was performed against whole-genome protein sequences of pear using the HMM profile (PF03195) of the LOB domain. Second, a local BLASTP analysis was performed to scan the whole-genome protein sequences of pear using the Arabidopsis thaliana LBD proteins as query sequences [15]. Initially, we retained the common protein sequences identified by both methods. To further verify the reliability of candidate $L B D$ genes, the SMART Tool, CDD tool and InterProScan tool were used to detect the completeness of the LOB domain of candidate proteins. As a result, four candidate genes with incomplete LOB domain were removed. Based on the location of candidate $L B D$ genes, we also removed five genes that were anchored on scaffolds. Consequently, a total of 60 non-redundant and complete $L B D$ genes were identified in the pear genome for further analysis. According to the presence/absence of the LX6LX3LX6L leucine zipper-like domain of LBD proteins, 60 PbrLBD genes were divided into two groups: 53 members belonged to class I and 7 members belonged to class II (Additional file 1: Figure S1). To distinguish members of the $P b r L B D$ gene family, we named each of $\operatorname{Pr} L B D$ gene based on their order on the chromosomes (Additional file 2: Table S1), namely, PbrLBD1-PbrLBD60. In addition, we further analyzed the physical and chemical properties of 60 LBD proteins. The ExPASy Proteomics Server, an online proteomics and sequence analysis tool, was used to predict the molecular weights and isoelectric points of $\operatorname{Pbr} L B D$ protein sequences. The length of $P b r L B D$-encoded protein sequences ranged from 150 (PbrLBD52) to 475 (PbrLBD7) amino acids. Protein masses ranged from $168.23 \mathrm{kD}$ (PbrLBD52) to $50.74 \mathrm{kD}$ (PbrLBD7), and protein pIs ranged from 4.56 (PbrLBD4) to 9.22 (PbrLBD13) (Additional file 2: Table S1).

Chromosome location of PbrLBD genes in the pear genome

To further investigate the distribution pattern of PbrLBD genes on chromosomes, we mapped all the members of the PrrLBD gene family in the pear genome. Mapchart 
software was used to show the location distribution of PbrLBD genes across the pear genome (Additional file 3: Figure S2). As a result, we found that $60 \mathrm{PbrLBD}$ genes were unevenly distributed in the pear genome, with the exception of chromosome 4, chromosome 13 and chromosome 16. Chromosome 5 contained the largest number of PbrLBD genes (8 members), followed by chromosome 1 and chromosome 15, which each contained $7 \mathrm{PbrLBD}$ genes. However, chromosome 6, chromosome 8 and chromosome 12 only contained one PbrLBD gene each. Three or more PbrLBD genes were mapped on chromosomes 2, 3, 7, 9, 10, 11, 14 and 17 . Interestingly, we found that most $P b r L B D$ genes existed in the form of gene clusters, suggesting that the PbrLBD family had undergone a WGD event/segmental duplication or tandem duplication.

\section{Phylogenetic analysis of the PbrLBD genes}

The phylogenetic tree is widely used to show the evolutionary relationship of gene families. A neighbourjoining (NJ) phylogenetic tree was constructed with the Mega-X program using the full-length LBD proteins of pear, apple and Arabidopsis (Fig. 1a). According to the phylogenetic tree results, a total of 168 LBD proteins from three species were phylogenetically categorized into seven subgroups, namely, class Ia, Ib, Ic, Id, Ie, and class IIa and IIb. In apple, 17, 6, 3, 8, 9, 2 and $12 M d L B D$ genes fell within the subgroups, respectively. In Arabidopsis, 7, 10, 3, 4, 9, 6 and 3 AtASL genes were clustered into the seven subgroups, respectively. In pear, class Ia and class Ib included the highest number of $P b r L B D$ genes (14), followed by class Ie, which contained 11 PbrLBD genes (Fig. 1b). This result provides further evidence that class I contains a greater percentage of $L B D$ genes than class II. It is important to note that most sister pairs of subclades consisted of PbrLBDs and MdLBDs (such as, PbrLBD35/MdLBD44, PbrLBD20/MdLBD26, PbrLBD14/ $M d L B D 16$, etc.), while most of the $A t L B D$ genes were separate from $\operatorname{PbrLBD}$ genes and $M d L B D$ genes (such as, ASL2, ASL25, ASL26 and ASL27). This result suggests that the $P b r L B D$ genes and $M d L B D$ genes had a closer evolutionary relationship, and consistent with the fact that apple and pear evolved from the same ancestor.

\section{Conserved motif analysis and gene structural analysis of PbrLBD genes}

To identify gene structures and evolutionary trajectories of $L B D$ genes in pear, we investigated exon-intron compositions of the $60 \mathrm{PbrLBDs}$. The number of introns of the $P b r L B D$ genes ranged from zero to four, suggesting that the introns of some PbrLBDs were lost during evolution. The PbrLBD7 gene contained five exons and four introns. In addition, the genes clustered into subclades had similar gene structures (Fig. 2a, c). Most members of class II had two exons and two UTR regions, whereas no UTR regions were detected for most members of class I.

The MEME (Multiple Em for Motif Elicitation) tool was used to predict the conserved motifs of PbrLBD proteins (Fig. 2b) [16]. A total of 20 conserved motifs were identified in our study, named motif $1-20$. As a result, the numbers and types of conserved motifs in PbrLBD protein sequences were relatively conserved. Most members clustered into the same subclade had similar motif structures, suggesting that proteins from the same subclade may have similar functions. Motif 1 and motif 2 were basic regions of the LOB domain that were detected in all members of the $\operatorname{PbrLBD}$ gene family. Class I members had motif 1 (CX2CX6CX3C), motif 2 (GAS blocks) and motif 3 (LX6LX3LX6L), while Class II members lacked motif 3 (LX6LX3LX6L). This result provided further evidence to support division of the PbrLBD gene family into two clusters.

\section{Cis-acting elements analysis in the putative promoter of PbrLBD genes}

In general, the gene function was influenced by the distribution of cis-acting elements in the promoter region [17]. In this study, the region $2000 \mathrm{bp}$ upstream (putative promoter sequences) of the transcription initiation site of $\operatorname{Pb} L B D$ genes was defined as the putative promoter region. The putative promoter sequences of $\operatorname{PbrLBD}$ genes were then submitted to the PlantCARE database to search for cisacting elements (Fig. 3) [18]. As a result, a total of 266 cis-acting elements for plant growth and development were identified, and we selected 11 important cis-acting elements for further analysis (Fig. 3). In Fig. 3a, a diversity of distribution patterns of cisacting elements was observed in the promoter region of $\operatorname{PbrLBD}$ genes, suggesting that the expression of $\operatorname{PbrLBD}$ genes may be regulated by various factors, such as, light, abscisic acid (ABA), methyl jasmonate (MeJA), gibberellin (GA) response element and myeloblastosis (MYB) binding site involved in drought-inducibility. For hormone-related elements, the ABA-responsive and MeJA-responsive elements were most frequently found in the pear LBD gene promotors. Previous studies had reported that four auxin-inducible $L B D$ genes ( $L B D 16 / A S L 18$, LBD17/ASL15, LBD18/ASL20 and LBD29/ASL16) were regulated by AUXIN RESPONSE FACTOR7 (ARF7) and ARF19 to control the formation of lateral root $[3,7]$. In our study, the IAA responsiveness element $(I X)$ were predicated on the putative promotor of $32 \mathrm{PbrLBD}$ genes, this result indicated that the functions of $P b r L B D$ genes might be regulated by auxin. It is notable that the element related 


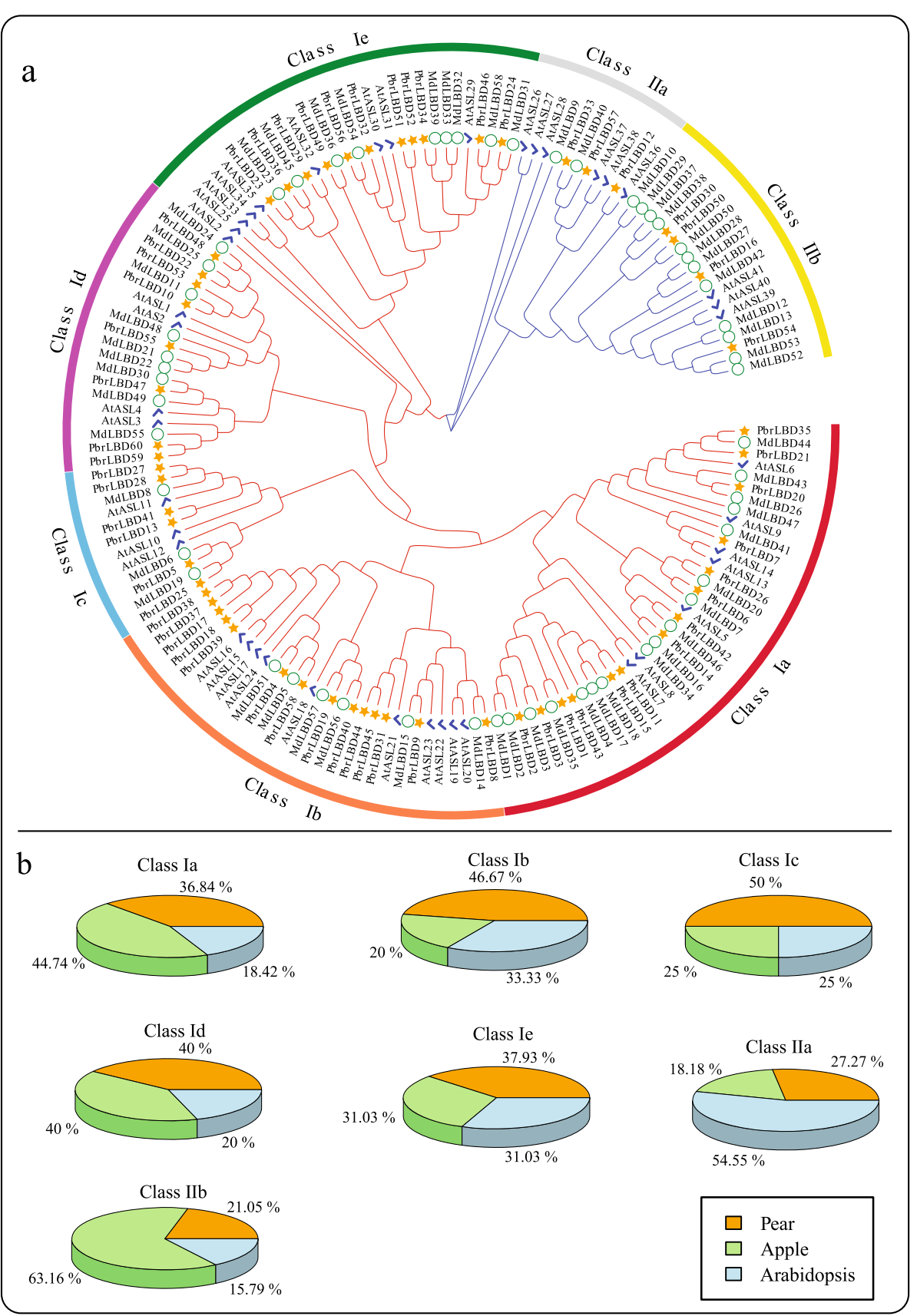

Fig. 1 Phylogenetic tree and the distribution of LBD genes of three species in seven subgroups, including class la-ld, class Ila and class IIb. (a) A phylogenetic tree of LBD proteins of pear, apple and Arabidopsis. A phylogenetic tree of LBD protein family was constructed by Mega-X using the Neighbor-Joining (NJ) method and 1000 bootstraps. The red branches indicates the class I genes, and the blue branches indicates the class II. The yellow star, green circle and blue tick indicates the LBD protein in pear, apple and Arabidopsis, respectively. (b) The percentage of $\angle B D$ genes of three species in each subgroup. The orange part indicates the proportion of $\angle B D$ genes in pear; The green part indicates the proportion of $\angle B D$ genes in apple; The blue part indicates the proportion of $\angle B D$ genes in Arabidopsis

to MYB binding site involved in flavonoid biosynthetic gene regulation was predicted in five $\operatorname{Pbr} L B D$ genes (PbrLBD5, PbrLBD17, PbrLBD49, PbrLBD51 and PbrLBD55), suggesting that they may play important functions in the process of flavonoid biosynthesis.

\section{Synteny analysis reveal the expansion of the $L B D$ gene family}

Previous studies have reported that the expansion of gene families is driven by different gene duplication modes, including whole-genome duplication (WGD)/ 


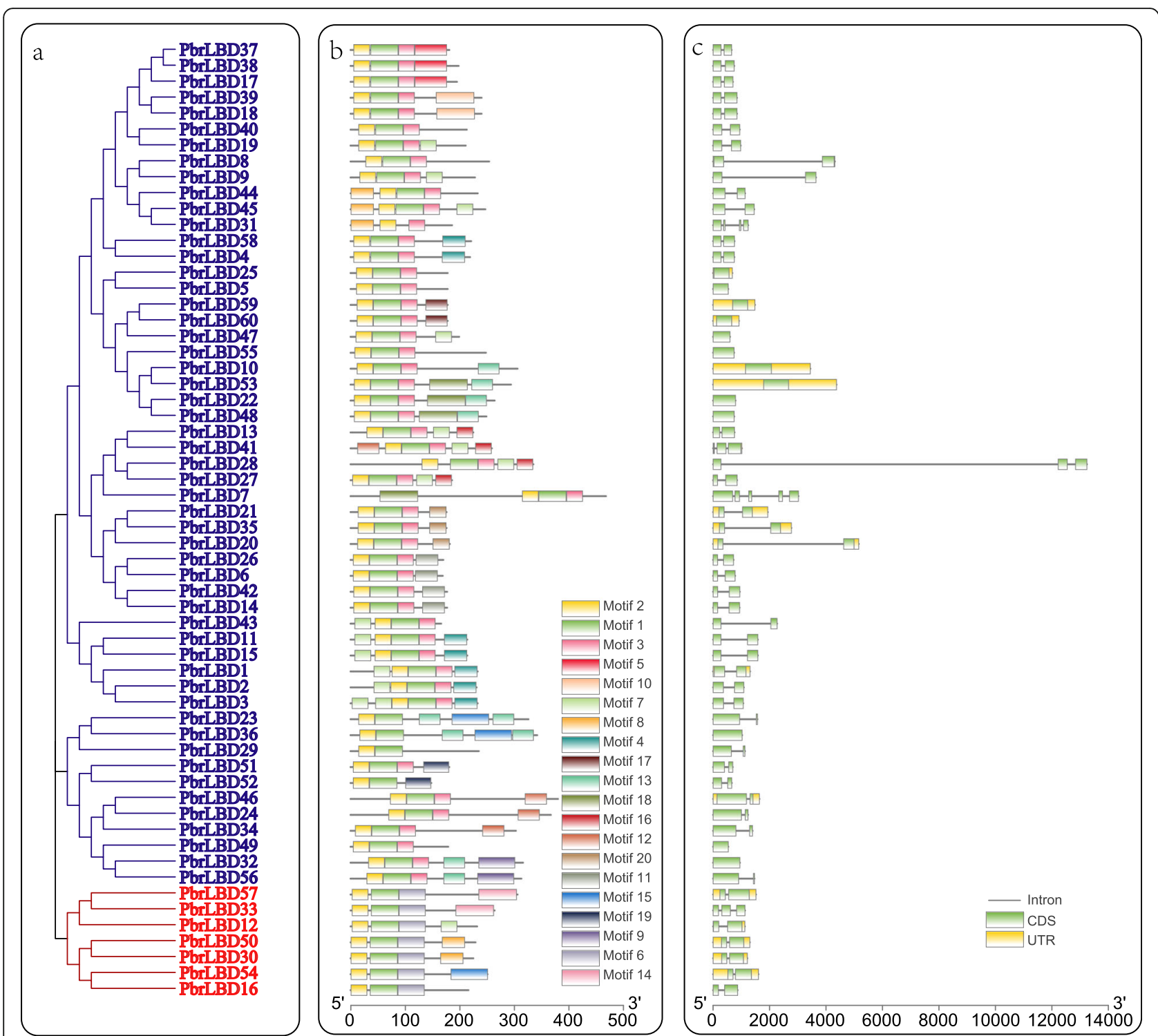

Fig. 2 The phylogenetic relationship, conserved motifs and gene structure of PbrLBD proteins. (a) A phylogenetic tree of PbrLBD protein family was constructed by Mega-X using the Neighbor-Joining (NJ) method and 1000 bootstraps. The blue branches indicate class I; the red branches indicate class II. (b) The distribution of conserved motifs across 60 PbrLBD protein. A total of 20 motifs were predicted using MEME (Multiple Em for Motif Elicitation) tool in our study. The scale bar indicates 100 aa. (c) The gene structure of PbrLBD genes, including intron, exon, UTR. The black line indicates intron; the green rectangle indicates exon; the yellow rectangle UTR. The scale bar indicates $2 \mathrm{~kb}$

segmental duplication, dispersed duplications (DD), tandem duplication (TD), proximal duplication (PD), and transposed duplication (TRD). To explore which duplication types drove the expansion of the PbrLBD gene family, a synteny analysis was conducted using the MCScanX software package (Fig. 4). Then, the DupGen finder tool was used to dissect gene duplication types with a priority of duplicate genes as follows: WGD > TD $>$ PD $>$ TRD $>$ DD. As a result, 58 PbrLBD genes were assigned to one of five different duplication types. Consequently, $76.67 \%$ (44) genes in the PbrLBD gene family were duplicated and retained from WGD/ segmental duplication types, followed by DD $(6,10 \%)$, TD (6, 10\%), PD (1,1.67\%) and TRD (1, 1.67\%) (Additional file 4: Figure S3). This result shows that lineagespecific WGD/segmental duplication events were the major force in the expansion of the PbrLBD gene family.

To further investigate the evolutionary mechanisms of the PbrLBD gene family, we performed a method described on the Plant Genome Duplication Database (PGDD) to identify the synteny blocks in the pear genome. A total of 24 synteny blocks containing PbrLBD genes were identified. The 22 duplicated gene pairs from the $\operatorname{PbrLBD}$ gene family were mapped in the 19 synteny 
a

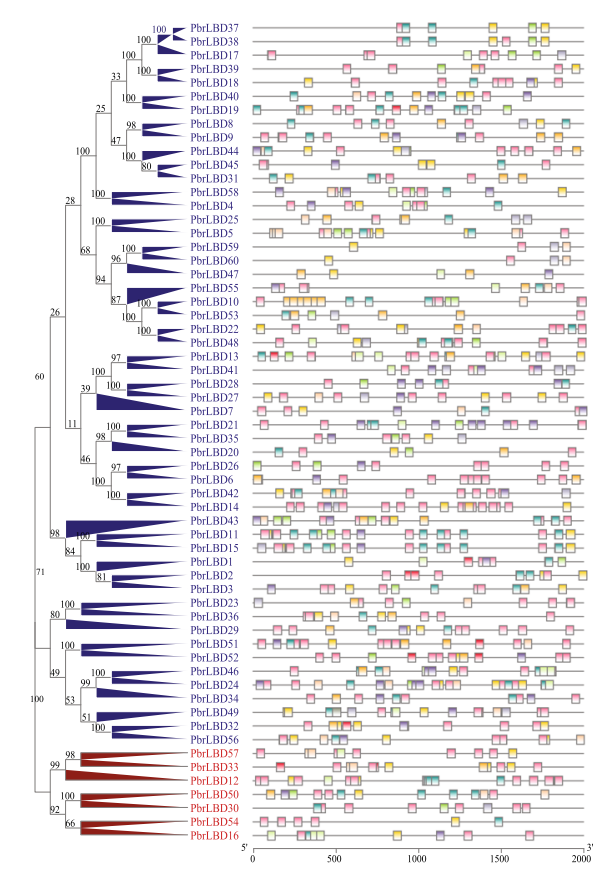

(IX) IAA responsiveness

(XI) GA responsive element

(II) light responsive element

(X) MeJA-responsiveness

(VI) ABA responsiveness

(IV) Defense and stress responsive element

(I) Meristem expression

(VII) Salicylic acid responsiveness

(V) MYB binding site involved in drought-inducibility (III) Low-temperature responsiveness

(VIII) MYB binding site involved in flavonoid biosynthetic genes regulation $\mathrm{b}$

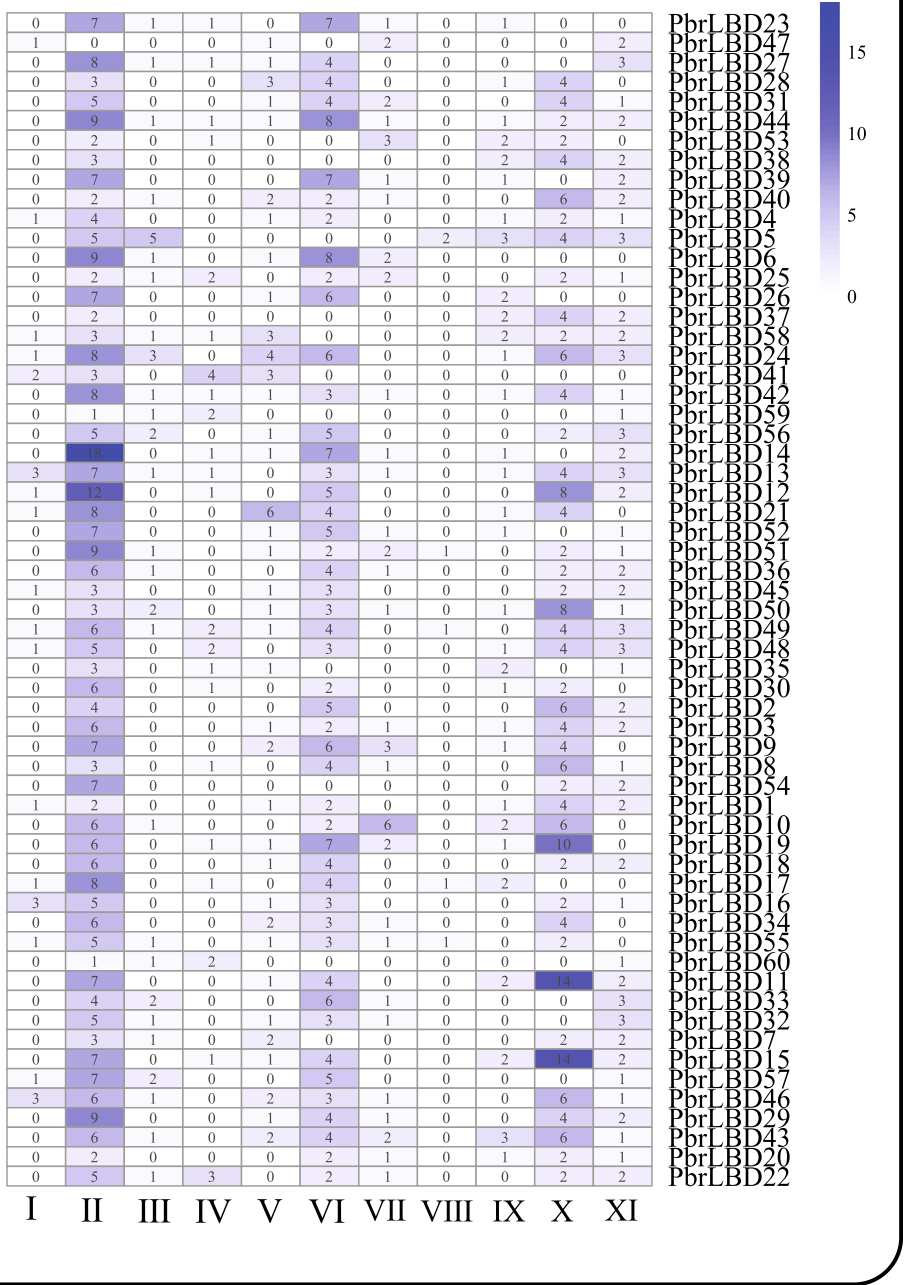

Fig. 3 The cis-acting elements on putative promoters of PbrLBD genes. (a) The distribution of cis-acting elements on putative promoters of PbrLBD genes. A phylogenetic tree of PbrLBD protein family was constructed by Mega-X using the Neighbor-Joining ( $\mathrm{NJ}$ ) method and 1000 bootstraps. The blue branches of phylogenetic tree indicate $\angle B D$ genes in class 1 ; the red branches of phylogenetic tree indicate $\angle B D$ genes in class II. (b) The number of cis-acting elements on putative promoters of PbrLBD genes. A total of eleven cis-acting elements were investigated in our study, including: (I) Meristem expression, (II) light responsive element, (III) Low-temperature responsiveness, (VI) ABA responsiveness, (V) MYB binding site involved in drought-inducibility, (VI) ABA responsiveness, (VII) Salicylic acid responsiveness, (VII) MYB binding site involved in flavonoid biosynthetic genes regulation, (IX) IAA responsiveness, (X) MeJA-responsiveness, (XI) GA responsive element

blocks, named synteny block 1-19 (Additional file 5: Table S2 and Fig. 4a). Among these synteny blocks, we found that several were strongly conserved. For example, synteny block 4 contained over 140 syntenic gene pairs. In order to further verify the reliability of synteny analysis, we investigated the distribution of the genes and the duplicated gene pairs in the 100-kb upstream and downstream regions of $\operatorname{Pbr} L B D$ genes (Additional file 6: Table S3 and Fig. 4b). We found that most PbrLBD genes were located in 200-kb conserved synteny blocks. These results provided further evidence that expansion of the PbrLBD gene family was mainly driven by a WGD/segmental duplication event.

\section{Estimating dates and driving forces for evolution of the PbrLBD gene family}

The number of synonymous substitutions per site (Ks) is extensively used to calculate the approximate occurrence dates of WGD/segmental duplication events. Our previous studies indicated that two WGD events had occurred during pear genome evolution, including an ancient WGD event $(\mathrm{Ks} \sim 1.5-1.8$, approximately 140 

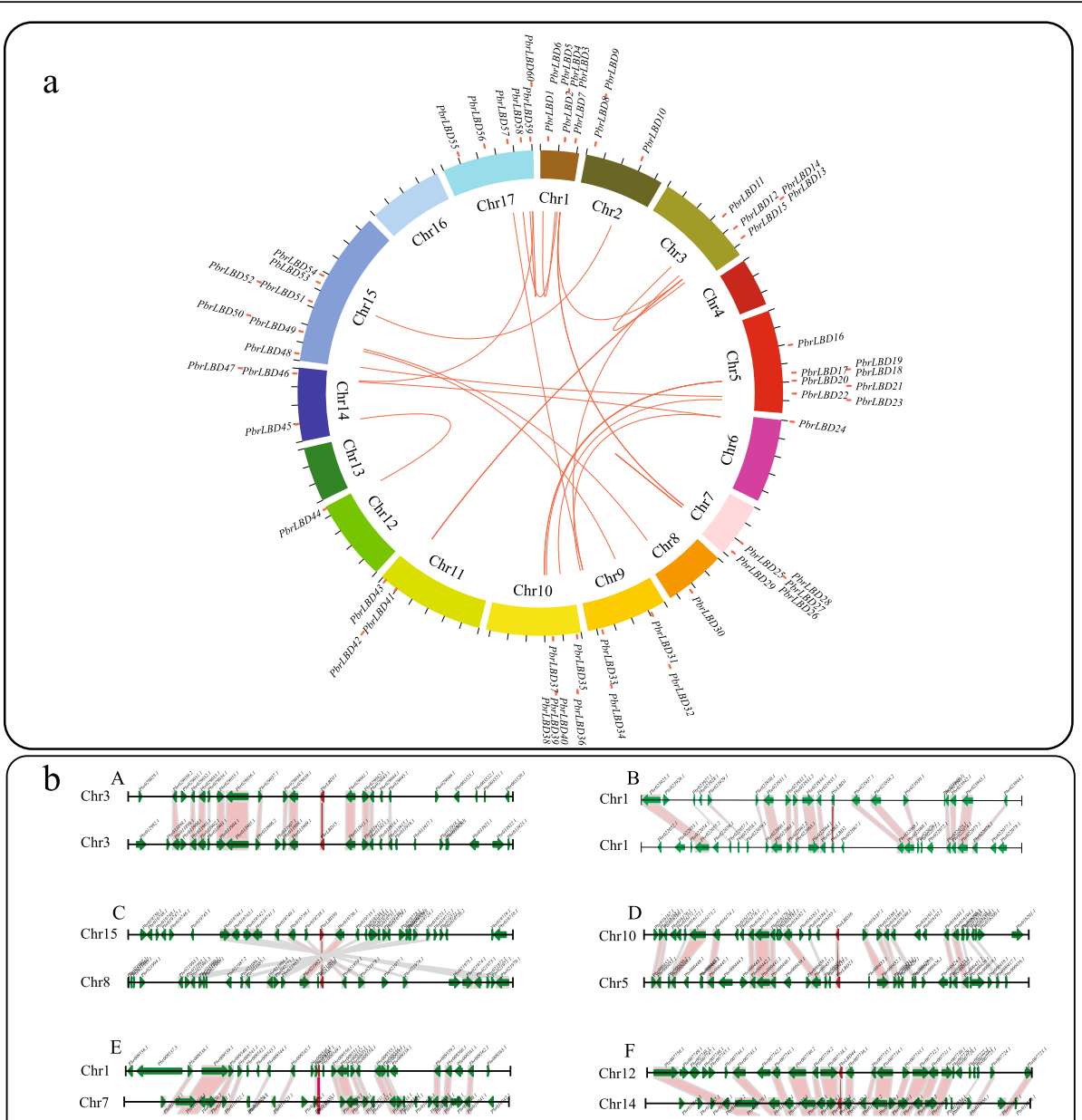

C
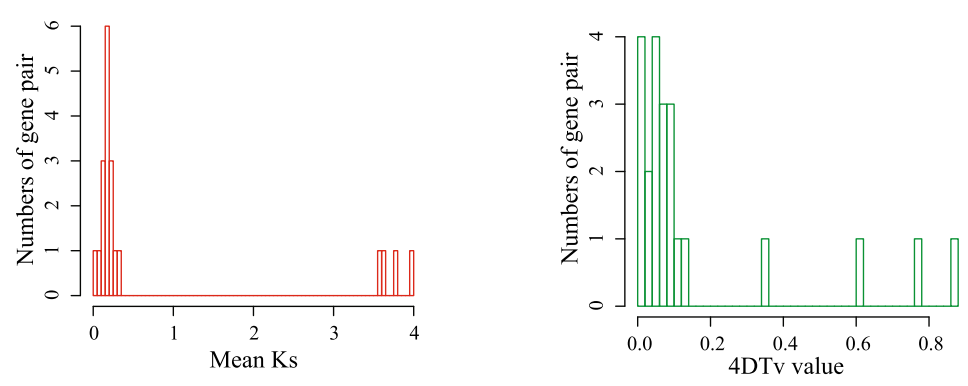

Fig. 4 The evolution analysis of PbrLBD gene family. (a) Distribution and synteny of PbrLBD gene family in pear genome. The circle picture was plotted by the Circos software (version 0.69.2). The red points mark the position distribution of $\angle B D$ gene on 17 pear chromosomes. The red lines show the collinear gene pairs of $\angle B D$ gene family. (b) Segmental duplication between members of PbrLBD family. (A) PbrLBD11 (Pbr029040.1) and PbrLBD15 (Pbr033910.1), (B) PbrLBD1 (Pbr033910.1) and PbrLBD2 (Pbr022066.1), (C) PbrLBD50 (Pbr019737.1) and PbrLBD30 (Pbr021980.1), (D) PbrLBD36 (Pbr016186.1) and PbrLBD23 (Pbr000434.1), (E) PbrLBD5 (Pbr009548.1) and PbrLBD25 (Pbr010928.1), (F) PbrLBD44 (Pbr007737.1) and PbrLBD45 (Pbr016876.1). The figure shows a region of $100 \mathrm{~kb}$ on each side flanking the PbrLBD genes. Duplicated gene pairs are connected with bands. Chromosome segments are indicated by black horizontal lines, and a broad line with arrowhead represents a gene and its transcriptional orientation. Text indicates the gene ID. The PbrLBD genes are shown in red, duplicated genes and other genes are shown in green; and homologous genes are linked by the red line. (c) The distribution of mean Ks and 4DTV value of 22 PbrLBD duplicated gene pairs. In the left figure, the $x$-axis shows the mean Ks value, and the $y$-axis shows the number of gene pairs. In the right, the $x$-axis shows the 4DTv value, and the $y$-axis shows the number of gene pairs. The data were plotted using the hist function in $R$ 
MYA) derived from a paleohexaploidization $(\gamma)$ event and a relatively recent WGD event (Ks $\sim 0.15-0.3$, approximately 30-45 MYA) [19, 20]. To further investigate the evolution dates $\operatorname{PbrLBD}$ gene duplication, mean Ks values of PbrLBD duplicated gene pairs were estimated to trace the date of $\mathrm{WGD} /$ segmental duplication events. Table S3 shows the mean Ks values for 22 PbrLBD paralogs in the syntenic region. The values ranged from 0.01 to 3.99. The gene sequences of several paralogs were completely consistent, which indicated that no mutations occurred between these gene pairs (PbrBD27/ PbrBD28, PbrBD11/PbrBD15). Most of the mean Ks values of the 22 paralogs fell in the range from 0.15 to 0.30 (Fig. 4), suggesting that the duplication of these gene pairs may have taken place during the relatively recent WGD event, approximately 30-45 MYA. However, the mean Ks value of $P b r L B D$ paralogs were not mapped to the region with values from 1.5 to 1.8 , suggesting that the PbrLBD gene family had not undergone the ancient WGD event $(\sim 140$ MYA). Surprisingly, three paralogs (PbrBD18/PbrBD40, PbrBD33/PbrBD12 andPbrBD34/ PbrBD24) exhibited relatively higher mean Ks values (3.61-3.98), suggesting that they duplicated during a more ancient duplication event.

Recently, $\mathrm{Wu}$ et al. reported that the pear genome underwent two significant groups of synteny blocks: a recent whole-genome duplication event (4DTv ([4-fold synonymous (degenerative) third-codon transversion]) $0.08)$ and an ancient WGD event $(4 \mathrm{DTv} \sim 0.5)[20]$. To test these suppositions, we calculated the $4 \mathrm{DT}$ v values of 22 paralogs using our in-house scripts. The distribution of 22 paralogs ranged from 0 to 0.86 (Fig. 4c), and most members exhibited a strong peak with a $4 \mathrm{DTv}$-value at approximately 0.08 . This result further supported the conclusions of our mean Ks value analysis, which indicated the PbrLBD gene family had undergone only one WGD event occurring 30-45 MYA.

Three DNA mutation types have been identified in genome evolution, including beneficial, neutral and harmful mutations [21]. In nature, most neutral and harmful mutations are eliminated through purifying selection, while beneficial mutations are retained during the evolution process. The accumulation of beneficial mutations is important for plants and animals to respond to a complex and changing environmental conditions [22]. To identify which selection pressures were driven by the evolution of $P b r L B D$ genes, the $\mathrm{ka} / \mathrm{ks}$ ratios of 22 PbrLBD paralogs were calculated using the Kaks_calculator tool. A ka/ks ratio equal to one indicates neutral selection, less than one indicates negative selection (purifying selection) and higher than one indicates positive selection (Darwinian selection). Results showed that all ka/ks ratios of PbrLBD paralogs were less than 1, suggesting that negative selection (purifying selection) was the primary driving force for evolution of the PbrLBD gene family.

\section{Expression analysis of PbrLBD genes by EST database}

Expression sequence tags (EST) is the part of cDNA sequence from different tissues, the length ranged from 300 to $1000 \mathrm{bp}$. One EST represents an expression gene at one time in one cell or tissues. Expression sequence tags (EST) can provide further evidence to support the accuracy of the members of gene family from transcription level $[23,24]$. To verify the accuracy of $\operatorname{PbrLBD}$ gene identification, a BLASTN search was performed to scan the 'Dangshansuli' pear expressed sequence tag (EST) database using all PbrLBD gene sequences as the query. A total of 54 EST hits were found for the PbrLBD gene family, with hits occurring for 33 of the genes (Evalue $<10 \mathrm{e}-10$, identity $>95 \%$, length $>200 \mathrm{bp}$ ). Three genes (PbrLBD21, PbrLBD35 and PbrLBD24) had the greatest numbers of EST hits, with four EST hits each. This result provided further evidence that the $L B D$ gene set is reliable. However, there were $27 \mathrm{PbrLBD}$ genes that did not hit any tags from the Chinese White pear EST database. This may be due to there has been little research on $L B D$ genes in pear.

\section{Expression patterns of PbrLBDs in different tissues of pear} Transcriptome sequencing analysis was conducted using transcriptome data from six different tissues of 'Dangshansuli' cultivar including stem, ovary, petal, sepal, fruit and leaf [25]. The RPKM (reads per kilobase per million) values were measured to represent the expression level of PbrLBD genes, and a value of RPKM equaling 0 represented that the gene had no expression in libraries. The expression patterns of $60 \mathrm{PbrLBD}$ genes were shown in Fig. 5 using heatmap.2, a function of the gplots package in R. Among $60 \mathrm{PbrLBD}$ genes, $11 \mathrm{PbrLBD}$ genes were expressed in all six different tissues, indicating that they have various roles in the development of different tissues. 51.67, 60.00, 48.33, 73.33, 40.00 and $35.00 \%$ of all PbrLBDs were expressed in six different tissues corresponding to stem, ovary, petal, sepal, fruit and leaf, respectively. Thirty-nine genes were expressed in at least one of six different tissues, although the transcript abundance of several genes was relatively lower for certain tissues. However, $10 \mathrm{PbrLBD}$ genes were not expressed in any of the six different tissues, suggesting that a portion of $P b r L B D s$ were functional redundancies. As shown in Fig. 1b, the genes in class Ib (46.67\%) and Ic (50\%) have substantial expansion in the pear genome. According to transcriptome data, we found that seven members in class Ib were not expressed in anyone tissues, suggesting that these PbrLBDs in class Ib were functional redundancies. Among class Ic, three $L B D$ transcription factors PbrLBD25, PbrLBD13 and PbrLBD5 


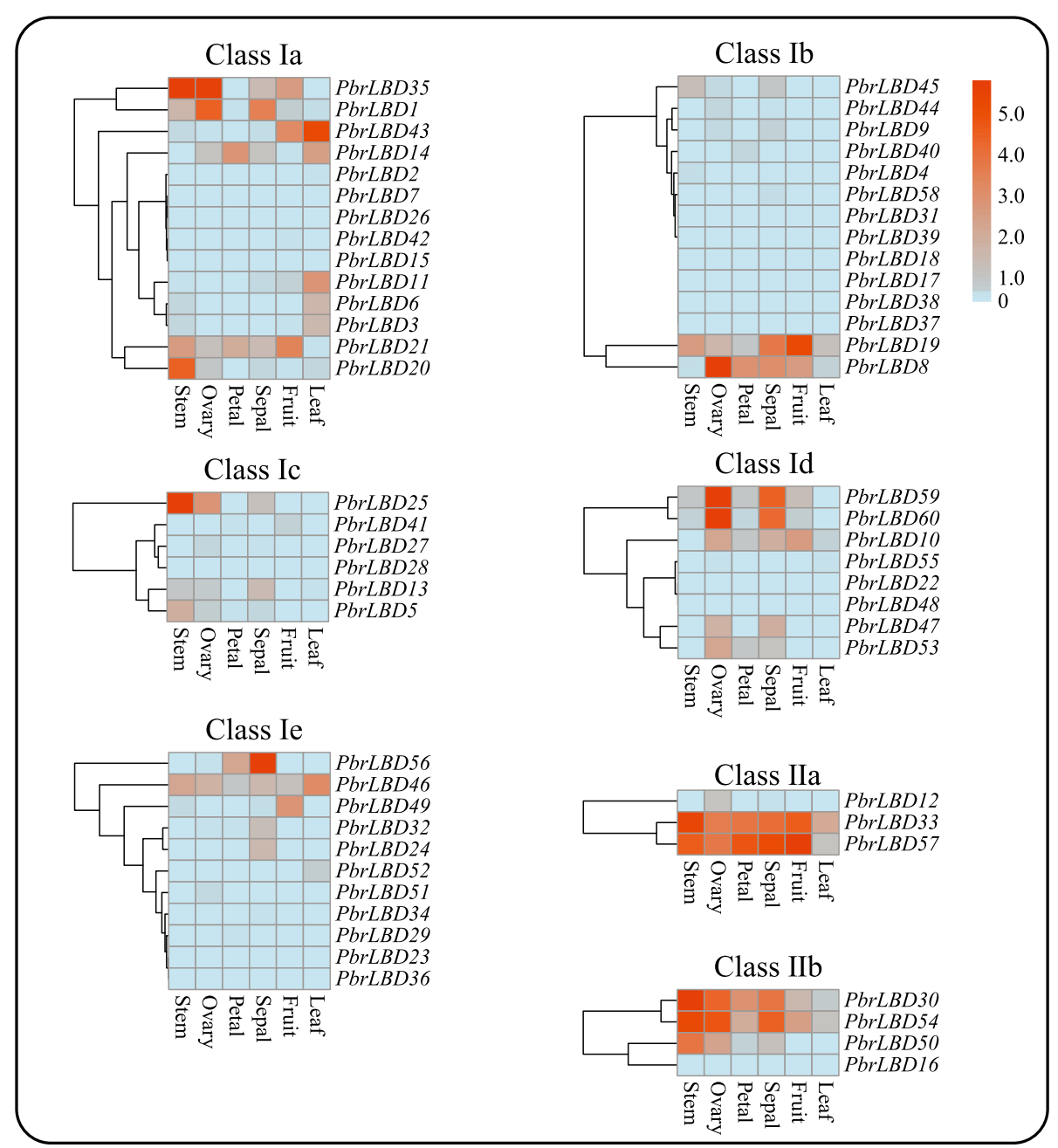

Fig. 5 Heatmap of expression level of 60 PbrLBD genes at six different tissues, including stem, ovary, petal, sepal, fruit and leaf. Based on the result of phylogenetic tree (Fig. 1a), the LBD gene family was divided into seven subgroups: class la, class Ib, class Ic, class Id, class Ila, class IIb. The heatmaps were plotted by heatmap.2, a function of the gplots package in R. Red color indicates high expression and blue indicates low expression. The color scale at the top right indicates RPKM (reads per kilobase per million) values normalized by log2

exhibited relatively high expression level in stem, suggesting that these three genes might occur neofunctionalisation. In summary, the result of transcriptome sequencing analysis supported that $\operatorname{PbrLBD}$ genes played important roles in multiple biological processes.

To verify that the functional cluster of transcriptome sequences analysis was reliable, 11 PbrLBD genes were selected to conduct a qRT-PCR experiment to investigate the expression levels in six different tissues of the 'Dangshansuli' pear, including stem, leaf, petal, fruit, sepal and ovary (Fig. 6). Results showed that expression of all 11 PbrLBD genes was detected in the six tissue types. Moreover, the $11 \mathrm{PbrLBD}$ genes exhibited a diversity of expression patterns in the six different tissues, suggesting that $P b r L B D$ genes may function in different tissues and participate in diverse metabolic processes. Previous studies had reported that $L B D$ genes strongly expressed in the micropylar side of the mature ovary [26]. In our study, seven genes (PbrLBD54, PbrLBD57, PbrLBD33, PbrLBD30, PbrLBD53, PbrLBD43 and PbrLBD50) exhibited a similar expression pattern with a high expression level in ovary tissues, suggesting that PbrLBD genes play critical functions during ovary development. The PbrLBD20 and PbrLBD25 genes were highly expressed in stem tissues, and $\operatorname{PbrLBD} 10$ genes were highly expressed in fruit. Overall, these PbrLBD genes may play critical roles in pear growth and development processes.

\section{Potential functions of PbrLBD genes in anthocyanin biosynthetic pathway}

Previous studies have revealed that some $L B D$ genes can act as important transcription factors in regulating anthocyanin accumulation $[2,27]$. To validate the 


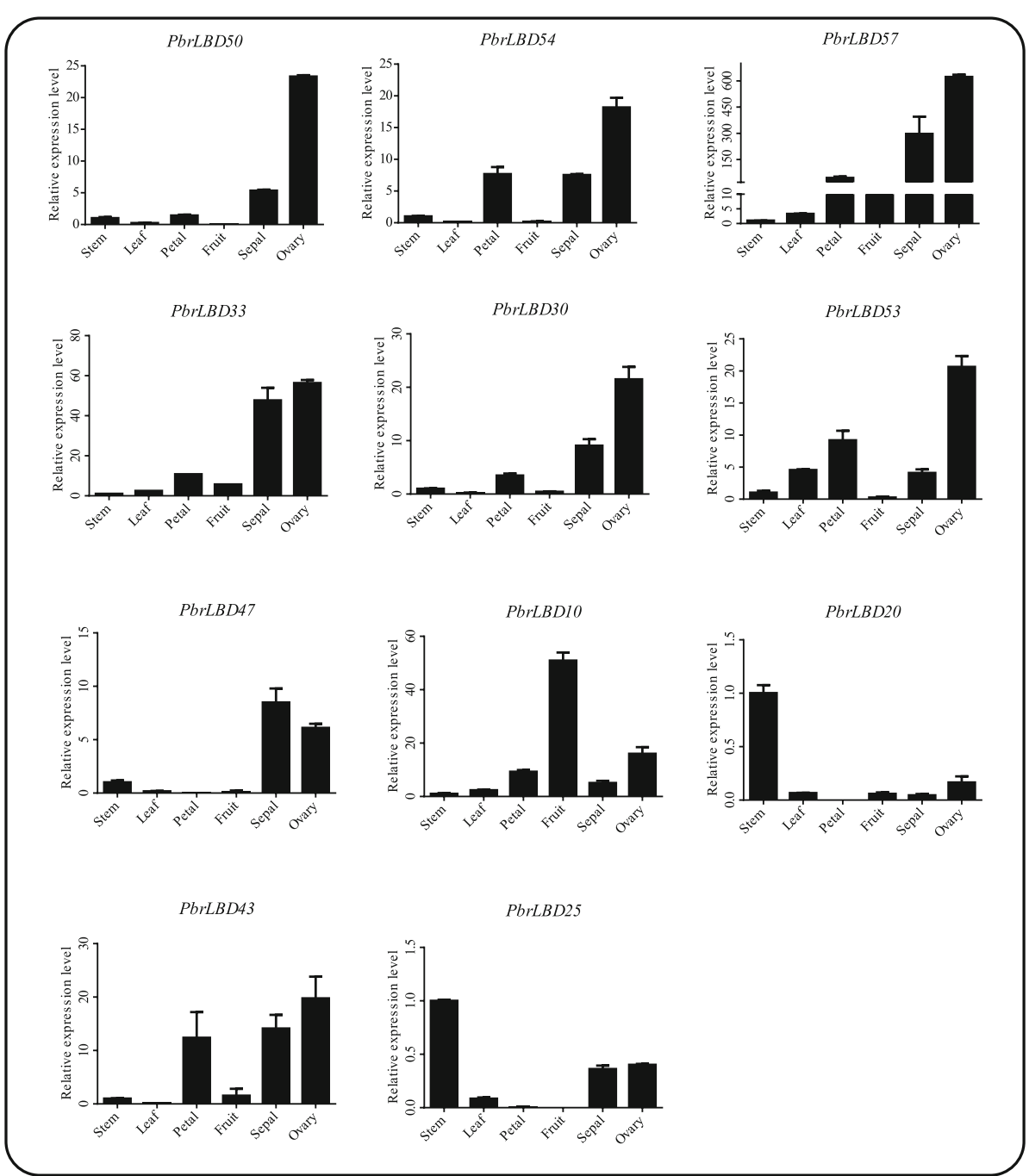

Fig. 6 Expression patterns of eleven PbrLBD genes at six different tissues, including PbrLBD50, PbrLBD54, PbrLBD57, PbrLBD33, PbrLBD30, PbrLBD53, PbrLBD47, PbrLBD10, PbrLBD20, PbrLBD43, PbrLBD25. Relative expression levels of PbrLBD genes were detected by qRT-PCR experiment. The x-axes represent six different tissues, including stem, ovary, petal, sepal, fruit and leaf; the $y$-axes represent relative expression level of $P b r L B D$ gene. Error bars indicate three technical replicates derived from one bulked biological replicate

potential functions of PbrLBDs in the anthocyanin biosynthetic pathway, we further analyzed our previous pear transcriptome data [28]. Compared to bagged fruits, the fruits removed from bags had significant higher anthocyanin concentrations in three comparisons (Fig. 7a). Differentially expressed genes (DEGs) analysis results indicated that four genes (PbrLBD20, PbrLBD33, PbrLBD35 and PbrLBD53) were differentially expressed in two or more comparisons of bagged fruit and fruit removed from bags (Fig. 7b). As shown in Fig. 7b, PbrLBD33 genes were found to be significantly upregulated in pears with high anthocyanin concentrations in all three comparisons. Furthermore, MYB binding sites involved in flavonoid biosynthetic gene regulation were found in the promotors of the PbrLBD33 gene. These results suggested that $\operatorname{PbrLBD} 33$ may participate positively in anthocyanin biosynthesis. However, expression of the other four DEGs was found to be downregulated in pears with high anthocyanin concentrations, suggesting that these genes may repress anthocyanin accumulation. These results provided strong evidence that these $L B D$ genes act as critical regulators in the anthocyanin biosynthetic pathway.

\section{Subcellular localization of PbrLBD20 protein}

In our study, the $L B D$ gene was a type of plant-specific transcription factor. PbrLBD2O was selected from four candidate anthocyanin-related genes for further subcellular localization, and its protein was predicted to be located in the nucleus. To observe the subcellular localization of PbrLBD20, a plasmid with the GFP gene fused to PbrLBD20 CDS and driven by the 35S promoter 
a
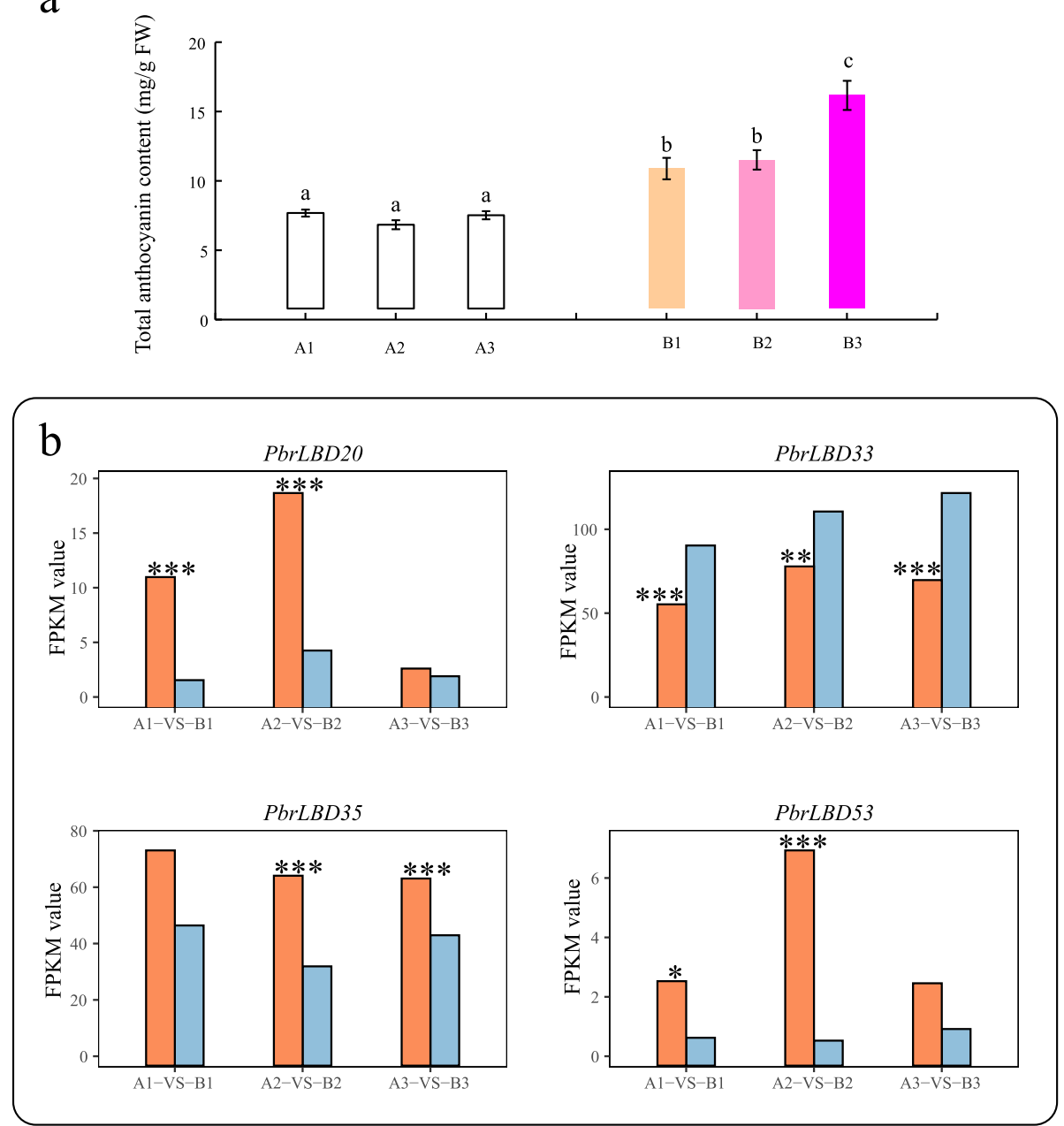

Fig. 7 The candidate PbrLBD gene associated with anthocyanin biosynthetic pathway. (a) Dynamic changes of total anthocyanin content (mg/g FW) in peels at different library. B1, B2 and B3 represent the fruits sampled at 4, 8 and 10 days after bag removal, and three bagged fruits (A1, A2, A3) also were sampled at the same time, as a control (Duncan test, $P<0.05$ ). (b) The FPKM (Fragments Per Kilobase per Million) values of four PbrLBD genes (PbrLBD20, PbrLBD33, PbrLBD35, PbrLBD53) related to anthocyanin biosynthetic pathway at three comparison, including A1-VS-B1, A2-VS-B2, A3-VS-B3. The $x$-axis represents the three different comparisons, and the $y$ - axis represents the FPKM values. Note: A-VS-B, orange bar represents $A$, light-blue bar represents $B\left({ }^{*} P<0.05,{ }^{*} P<0.01\right.$ and ${ }^{* *} P<0.001, T$-test $)$

was translocated into tobacco leaves. A confocal microscope was used to determine the localization of recombinant PbrLBD20. In Fig. 8, we observe that CK (empty vector) is located at the membrane and the nucleus, and PbrLBD20-GFP shows strong signals in the nucleus (Fig. 8). This result validates that $\mathrm{PbrLBD2O}$ is a transcription factor.

\section{Discussion}

\section{Whole-genome identification and phylogenetic analysis} of $\angle B D$ genes in pear

As plant-specific transcription factors, $L B D$ genes encode a conserved LOB (lateral organ boundaries) domain and are functional in diverse biological processes, including abiotic stress responses, anthocyanin biosynthesis, nitrogen metabolism and lateral organ development. Given their important function during plant development, $L B D$ genes have been identified in different plant species, such as apple (58) [11], rice (35), maize (44) [12], Arabidopsis thaliana (42) [5] and grape (40) [13]. Pear is one of the most important fruit trees, however, research on $L B D$ genes in pear has been relatively rare. In our study, a relatively strict criterion was performed to determine candidate members of the $L B D$ gene family in pear. The candidate genes lacked a conserved LOB domain, and genes that were unanchored on a chromosome were removed from consideration. With these criteria, a total of $60 \mathrm{PbrLBD}$ genes were identified, which is similar to the number identified for apple (58) [11]. According to the presence/absence of a conserved LX6LX3LX6L leucine zipper-like domain in the C terminus, members of the $L B D$ gene family were divided 


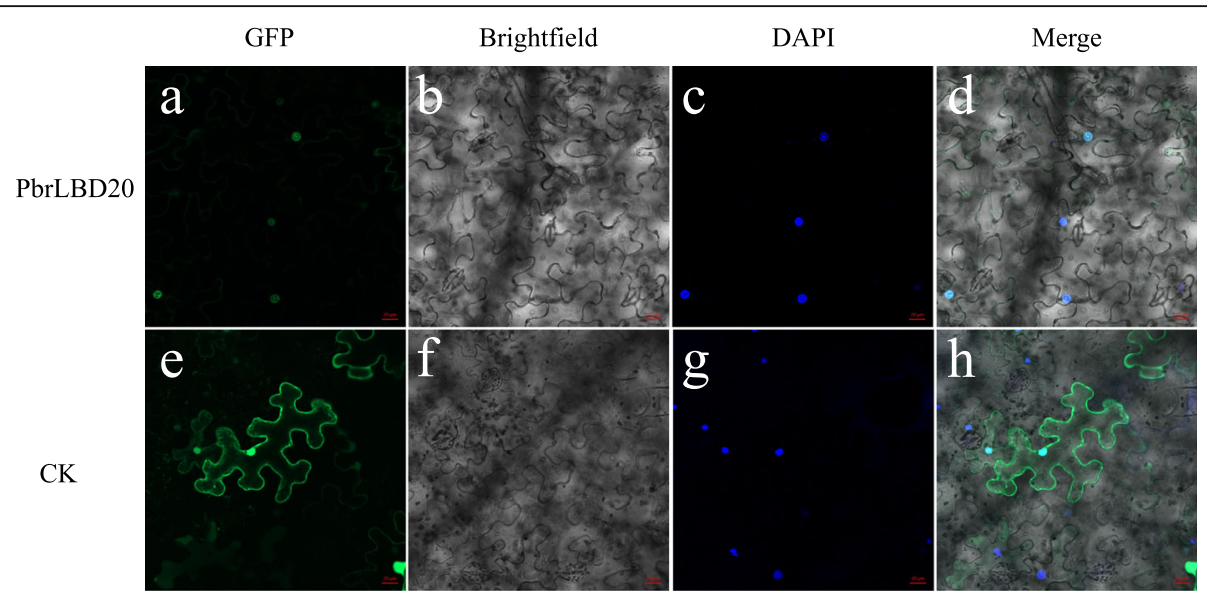

Fig. 8 Subcellular localization of PbrLBD20. PbrLBD20 was nuclear localized as determined by 35S:PbrLBD20-GFP fusion protein in tobacco leaf epidermal cells. Transient expression of GFP alone (35S:GFP) in tobacco leaf epidermal cells was used as a control (CK). The nucleus was identified by DAPI staining were used as markers. Images were taken in a dark field for green fluorescence, while the outline of the cells and the merged image were recorded in a bright field. Scale bars $=20 \mu \mathrm{m}$

into two classes (Fig. 1). Among them, 53 PbrLBD genes were classified as class I and six were classified as class II (Additional file 7: Figure S4). Previous studies had reported that $84 \%$ of members of the LBD gene family that were found in Arabidopsis and 78\% of those found in apple belonged to class I $[8,11]$. This result provided further evidence that a higher percentage of $L B D$ genes found in different species fall into class I versus class II.

Based on the phylogenetic grouping relationships, a total of 159 LBD genes in three different species were organized into seven major subgroups (Ia, Ib, Ic, Id, Ie, IIa and IIb). Although there are differences, this phylogenetic tree was largely consistent with the phylogenetic tree results from a previous study $[8,11]$. Homologous genes with similar functions typically will be clustered into the same subclades, which provides a valuable reference to predict gene function [12] (Fig. 1). Previous studies have reported that class II genes may play crucial roles in anthocyanin biosynthesis [12]. For example, one study found that ASL39/LBD37, ASL40/LBD38 and $A S L 41 / L B D 39$ could negatively regulate anthocyanin biosynthesis by responding to Nitrogen $(\mathrm{N})$ and nitrate $\left(\mathrm{NO}^{-}\right)$[2]. Therefore, we speculated that class II $L B D$ genes of pear might play important roles in anthocyanin biosynthesis.

\section{The evolution history of PbrLBD gene family}

Previous studies have reported that the expansion of gene families were mainly driven by gene duplication, such as WGD event/segmental duplication, and dispersed and tandem duplication [29, 30]. For example, the expansion of $A P 2 / E R F$ and WRKY gene families were primarily driven by WGD/segmental duplication and tandem duplications [31, 32]. The NBS-LRR gene family is thought to have expanded by transposed duplications [33]. There are at least two WGD/segmental duplication events that occurred in the evolution process of the pear genome, including an ancient WGD event (approximately $\sim 140$ MYA) and a relatively recent WGD event (approximately 30-45 MYA) [19, 34]. Synteny analysis indicated that WGD event/segmental duplication played a leading role in the expansion of the $\operatorname{PbrLBD}$ gene family. However, our results of mean Ks and $4 \mathrm{DTv}$ values of PbrLBD paralogs showed that the PbrLBD gene family had undergone only one recent WGD/segmental duplication event (30-45 MYA) [20]. It is interesting to note that five duplicated gene pairs (PbrBD60/PbrBD59, PbrBD14/PbrBD42, PbrBD26/ PbrBD6, PbrBD57/PbrBD33 and PbrBD13/PbrBD41) exhibited relatively low mean Ks values, ranging from 0.008 to 0.14 , suggesting that a more recent gene duplication event occurred in the evolution process of the PbrLBD gene family. Following duplication, duplicated gene pairs can undergo three different selection pressures, including positive selection, negative selection (purifying selection) and neutral selection [35]. Our analysis indicated that the evolution of the PbrLBD gene family was mainly driven by purifying selection.

\section{The potential roles of Chinese white pear $L B D$ transcription factors}

Increasing evidence indicates that $L B D$ genes play diverse roles during plant development processes. In general, the gene expression patterns can provide important clues to predict the gene function [36]. Transcriptome sequencing analysis and qRT-PCR expression profiling were conducted to investigate $L B D$ gene expression patterns in six different tissues. The members of the LBD 
gene family exhibited a variety of expression patterns in six different tissues, even in a single subclass. This result indicated that $\operatorname{PbrLBD}$ genes may participate in various biological processes. Ten genes were expressed in all tissues, suggesting these genes play a broad role in pear growth and development. Additionally, 73.33\% of PbrLBDs were detected in transcriptional abundance in pear sepal, suggesting these genes may have important roles in the development of pear sepal. Further, the expression levels of 11 randomly selected members of the PbrLBD gene family were validated using qRT-PCR. Based on qRT-PCR expression profiles, we found that most of the $11 \mathrm{PbrLBD}$ genes were highly expressed in flower organ, such as petal, sepal and ovary. Therefore, we predicted that $L B D$ genes may function in reproductive organs. A previous study reported that AtASL1 genes were involved in flower development through controlling the cell fate determination in Arabidopsis petals [37]. As shown in Fig. 1a, PbrLBD10 and PbrLBD53 were grouped into the same subclades with AtASL1, and exhibited relatively high expression levels in pear petals. This result indicated that $\operatorname{PbrLBD} 10$ and $\mathrm{PbrLBD} 53$ play an important role in petal development. Interestingly, we found that $\operatorname{PbrLBD10}$ was highly expressed in mature fruit. In grape, the expression levels of the two $L B D$ genes (GSVIVT01008284001 and GSVIVT01024592001) were low expressed before the fruit color change period, while were gradually increased during fruit ripening [38]. In banana, the expression level of MaLBD1, MaLBD2 and $M d L B D 3$ were increased during fruit ripening, and these three genes involved in regulating fruit ripening by activating the transcription of cell wall-loosening-related genes MaEXPs [39]. Similarly, we speculated that PbrLBD10 gene might involve in the regulation of fruit ripening and related color changes. However, the function of $L B D$ genes in fruit is still unknown and needs to be investigated further in future studies.

Anthocyanins are important flavonoid compounds detected in plants that are beneficial to human health [40, 41]. In pear, anthocyanin concentration determines fruit quality and color. However, anthocyanin accumulation is influenced by many environmental conditions, such as temperature, light and drought [42]. Numerous studies have indicated that $L B D$ genes play important roles in anthocyanin biosynthesis processes. In apple, the overexpression of $M d L B D 13$ negatively regulated anthocyanin accumulation through repressing the expression levels of structural genes in the flavonoid pathway [27]. In Arabidopsis thaliana, ASL39/LBD37, ASL40/LBD38 and ASL41/LBD39 negatively regulated anthocyanin biosynthesis in response to $\mathrm{N} / \mathrm{NO}_{3}{ }^{-}[8]$. In this study, transcription sequencing data from pear fruits after bagging and bag removal indicated that PbrLBD20, PbrLBD33, PbrLBD35 and PbrLBD53 may act as important regulators in anthocyanin accumulation. Interestingly, the light-responsive elements were found in the promoters for all four of these genes. This result indicated that these four PbrLBD genes may be involved in light-responsive anthocyanin biosynthesis.

\section{Conclusions}

In our study, a total of $60 \mathrm{LBD}$ genes were identified in pear genome, with an unevenly distributed among chromosomes. According to gene structure and phylogenetic analysis, the PbrLBD gene family was divided into two classes: class I (53) and class II (7). Synteny analysis further indicated that WGD/segmental duplication (30-45 MYA) played a key role in the expansion of $\operatorname{PbrLBD}$ gene family. Purifying selection was primary force during PbrLBD gene family evolution process. Based on mean Ks values and $4 \mathrm{DTV}$ values, PbrLBD gene family undergone only one recent WGD event occurring at 30-45 MYA. Transcription sequencing data from pear fruits after bagging and bag removal further indicated that PbrLBD33 may positively regulate the anthocyanin accumulation, while genes PbrLBD20, PbrLBD35 and PbrLBD53 act as negative roles. Interestingly, the light-responsive elements were found in the promoters for all four of these genes. This result indicated that these four PbrLBD genes may be involved in light-responsive anthocyanin biosynthesis. Further functional analysis indicated $\mathrm{PbrLBD} 20$ was localized in the nucleus. These results provide valuable information for understanding the evolution of the PbrLBD gene family, and facilitate further research on functional characterization of $\mathrm{PbrLBD}$ genes in anthocyanin biosynthesis in future studies.

\section{Methods}

\section{Whole-genome identification of $L B D$ gene family in Chinese white pear}

The pear (Pyrus bretschneideri) genome sequence was downloaded from the pear genome project (http://peargenome.njau.edu.cn/) [20] and the Arabidopsis LBD protein sequences were downloaded from the Plant Transcription Factor Database (PlantTFDB) (http:// planttfdb.cbi.pku.edu.cn). First, all pear protein sequences were scanned by BLASTP using all Arabidopsis LBD protein sequences as queries [12]. Second, we performed a hidden Markov model search (HMMsearch) for the pear protein database using the HMM profile with the LOB domain (PF00847) [8]. The redundant sequences between the results of HMMsearch and BLASTP were removed. Then, the CDD tool (https:// www.ncbi.nlm.nih.gov/cdd), SMART tool (http://smart. emblheidelberg.de/) and InterProScan tool (http:// www. ebi.ac.uk/Tools/pfa/iprscan/) were used to verify the completeness of the LOB domain. The distribution of identified $L B D$ genes across chromosomes were extracted 
from the pear genome database using an in-house Python script, and displayed in Figure S1 by using MapChart [43]. In additional, we predicted the protein molecular weights and isoelectric points of pear $L B D$ genes on the ExPASy proteomics server, an online proteomics and sequence analysis tool (http://expasy.org) [44].

\section{Gene structure and conserved motif analysis}

Gene structural information for PbrLBD genes was obtained from the pear genome database and displayed using TBtools software [45]. The MEME tool (http:// meme-suite.org/tools/meme) was used to identify the conserved domain motif by multiple alignment analysis [46]. The parameters were set as following: repetitions, any number; number of different motifs, 20; minimum motif width, 30; maximum motif width, 70. ClustalW analysis was performed using the full-length protein sequences of PbrLBD genes with default multiple alignment parameters and visualization was achieved with GeneDoc software [47, 48].

\section{Multiple sequence alignment and phylogenetic analysis} The construction of the phylogenetic tree followed a two-step process. First, all LBD full-length protein sequences in Pyrus bretschneideri, Arabidopsis thaliana and Malus domestica were aligned using ClustalW with default parameters [47]. Then, an unrooted phylogenetic tree was constructed by MEGA-X based on the result of multiple sequence alignment using the NJ (NeighborJoin) method with 1000 bootstrap replicates [49]. Evolview (version 2) [50], an online visualization tool, was used to visualize the phylogenetic tree.

\section{Cis-regulatory elements analysis of $P b r L B D$ gene promoters}

Bedtools software was used to extract the 2000-bp upstream (putative promoter region) sequences of the transcription start site of all PbrLBD genes [51]. Then, PlantCare was used to predict the cis-acting elements in the putative promoter region of $\mathrm{PbrLBD}$ genes [18].

\section{Synteny analysis of PbrLBD genes}

We performed synteny analysis to identify synteny blocks using a method described in the PGDD database (http://chibba.agtec.uga.edu/duplication/). First, a BLASTP alignment was conducted to search for candidate homologous gene pairs across the whole genome of Chinese White pear, and the parameters were set to: Evalue, 1e-10; -max_target_seqs, 5 [52]. To identify syntenic chains, these candidate homologous gene pairs served as the input for MCScanX software (default parameters) [53]. DupGen_finder-unique was further used to distinguish the whole-genome duplication (WGD) /segmental duplication, single duplication (SD), tandem duplication (TD), proximal duplication (PD), and transposed duplication (TRD), with the priority of the duplication genes as follows: WGD $>$ TD $>$ PD $>$ TRD $>$ DD [54]. The synteny and position information of $L B D$ genes was displayed by plotting a graph using Circos software (version 0.69.2).

\section{Calculating $\mathrm{Ka}$, Ks and 4DTv of PbrLBD paralogs}

ParaAT 2.0 [55] and Kaks_calculator 2.0 [56] were used to calculate the $\mathrm{Ka}, \mathrm{Ks}$ values of $\mathrm{PbrLBD}$ paralog gene pairs located in the same synteny block. The Kaks_calculator 2.0 parameters were set as follows: $\mathrm{YN}$ as the Method $(-\mathrm{m})$ and Standard Code as the Genetic code table $(-\mathrm{c})$. 4DTv (4-fold synonymous third-codon transversion) was widely used to estimate the genetic distances of synteny gene pairs. We calculated 4DTv values of PbrLBD paralogs using our in-house Python script. The distribution of mean Ks and 4DTv values of 22 paralogs were plotted using the hist function in $\mathrm{R}$.

\section{Expression analysis by ESTs}

A local BLASTN alignment against Chinese white pear EST libraries was conducted to find the corresponding records for each member of the $\operatorname{PbrLBD}$ gene family using the following parameters: maximum identity > $95 \%$, length $>200 \mathrm{bp}$, and E-value $<10^{-10}$.

\section{Expression pattern analysis of PbrLBD genes by transcriptome sequencing}

The RNA-seq data in six different tissues of the cultivar 'Dangshansuli' were acquired from our previous study, including stem, leaf, sepal, petal, fruit and ovary [57]. The RNA-seq data from sand pear cultivar 'Mantianhong' (Pyrus pyrifolia) samples comparing bagging and removal from bags also was acquired from our previous study [28]. We bagged the fruits at 35 days after full bloom (DAFB), and then bag removal at 10 days before commercial maturity. B1, B2 and B3 represent the fruits sampled at 4, 8 and 10 days after bag removal, and three bagged fruits (A1, A2, A3) also were sampled at the same time, as a control. We downloaded them from the National Center for Biotechnology Information database (NCBI, https://www.ncbi.nlm.nih.gov/). Heatmap.2, a function in $\mathrm{R}$, was used to plot heatmaps based on the $\log 2(\mathrm{RPKM}+1)$ value of each $\mathrm{PbrLBD}$ gene.

The measurement of anthocyanins content Anthocyanins were extracted and measured according to the method described by previous study [58].

\section{Quantitative real-time PCR analysis (qRT-PCR)}

Samples of the six different tissues in "Dangshansuli" including stem, leaf, petal, fruit, sepal and ovary were collected for qRT-PCR analysis. Total RNA was 
extracted from mixed samples of the six different tissues using a Plant Total RNA Isolation Kit Plus (FOREGENE Co. Ltd., Chengdu, China). Then, total RNA concentrations were adjusted to all be the same, and then first-strand cDNA was synthesized using TransScript One-Step gDNA Removal and cDNA Synthesis SuperMix (TransGen Biotech Co. Ltd., Beijing, China). Eleven pairs of the most specific primers were designed by using NCBI online software (https://www.ncbi.nlm.nih.gov/tools/primerblast), which amplified the 11 candidate gene sequences (Additional file 8: Table S4). qRT-PCR analysis was performed using LightCycler 480 SYBR GREEN I Master (Roche). A 20- $\mu$ l mixed reaction system was set up. Each sample contained $150 \mathrm{ng}$ of template cDNA, $0.5 \mu \mathrm{M}$ of each primer pair and $10 \mu \mathrm{l}$ of LightCycler 480 SYBR GREEN I Master. All reactions were set up in 96-well plates and each cDNA sample had three replicates. We set the qRT-PCR conditions as follows: first $5 \mathrm{~min}$ at $95^{\circ} \mathrm{C}$ for pre-incubation, $55 \mathrm{cy}$ cles at $95^{\circ} \mathrm{C}$ for $3 \mathrm{~s}, 60^{\circ} \mathrm{C}$ for $10 \mathrm{~s}$, and $72^{\circ} \mathrm{C}$ for $30 \mathrm{~s}$, and then $3 \mathrm{~min}$ at $72^{\circ} \mathrm{C}$ for extension. Finally, fluorescence signal data collection was carried out at $60^{\circ} \mathrm{C}$. Pyrus TUB (No. AB239681) was used for internal control genes (Additional file 8: Table S4). The average threshold cycle $(\mathrm{Ct})$ for each cDNA sample was calculated using the running results displayed on the computer. At the same time, the relative expression levels of the seven genes were calculated using the $2^{-\Delta \Delta \mathrm{Ct}}$ method, which is described in the previous study [59].

\section{Subcellular localization analysis}

For subcellular localization analysis of PbrLBD20, a 35SPbrLBD20-GFP fusion vector was constructed according to Xue et al. CE Design v1.04 (Vaezyme, China) was used to design specific primers for cloning and recombination [60]. Agrobacterium transformation (Agrobacterium tumefaciens strain GV3101) was conducted using the freeze-thaw method after the fusion vector was confirmed by sequencing. The empty GFP vector was used as a control. Then, the agrobacterium suspensions $\left(\mathrm{OD}_{600}=0.8 \sim 1.0\right)$ were injected into the abaxial surfaces of 3-week-old Nicotiana benthamiana plant leaves. After three days of culturing in a greenhouse, the injection sites and their surrounding tissues were collected for vacuum treatment and immediately stained with DAPI. Then, a Leica TCs SP2 spectral confocal microscope (Leica Microsystems, Germany) was used to observe green fluorescence and nuclear DNA signals. ZEN 2012 (Carl Zeiss Microscopy GmbH, Germany) and Adobe Illustrator 2020 (Adobe Systems, USA) were used for image processing.

\section{Statistical analysis}

Anthocyanin content were analyzed with SPSS 20 software (IBM, USA). One-way ANOVA followed by Duncan test was used to compare the statistical difference at the significance level of $P<0.05$. The $T$-test were conducted to evaluate the significant differences of gene expression level between bagged and bag removal treatment $\left({ }^{*} \mathrm{P}<0.05,{ }^{* *} P<0.01\right.$ and $\left.{ }^{* * *} P<0.001\right)$.

\section{Supplementary information}

Supplementary information accompanies this paper at https://doi.org/10. 1186/s12864-020-06999-9.

Additional file 1: Figure S1. Conserved domains of PbrLBD protein family. (a) Multiple sequence alignment of PbrLBD proteins by ClustalW. The result of multiple sequence alignment was visualized by GeneDoc tool. (b) The logos of the $\mathrm{CX} 2 \mathrm{C} \times 6 \mathrm{C} \times 3 \mathrm{C}$ zinc finger-like domain and the LX6LX3LX6L leucine zipper-like domain.

Additional file 2: Table S1. The members of PbrLBD gene family in Chinese White Pear genome.

Additional file 3: Figure S2. The location of $P b r L B D$ genes on pear chromosomes.

Additional file 4: Figure S3. The number of $\angle B D$ genes in five duplication types.

Additional file 5: Table S2. The synteny block contained PbrLBD duplicated gene pairs.

Additional file 6: Table S3. The synteny analysis of $P b r L B D$ gene family. Additional file 7: Figure S4. The percentage of $\angle B D$ genes of each class in three species, including apple, pear and Arabidopsis.

Additional file 8: Table S4. The eleven pairs of primer sequences for amplifying PbrLBD genes by qRT-PCR analysis.

\section{Abbreviations}

LBD: Lateral organ boundaries domain; TF: Transcription factor; pl: Isoelectric point; WGD: Whole genome duplication; DD: Dispersed duplications; TD: Tandem duplication; PD: Proximal duplication; TRD: Transposed duplication; GA: Gibberellin; ABA: Abscisic acid; MeJA: Methyl Jasmonate; MYB: Myeloblastosis; 4DTv: 4-fold synonymous (degenerative) third-codon transversion; MYA: Million years ago; qRT-PCR: Quantitative real time polymerase chain reaction; DEGs: Differentially expressed genes

\section{Acknowledgments}

Not applicable.

\section{Authors' contributions}

BS carried data analysis and figure preparation. ZT conducted the qRT-PCR and subcellular localization experiments. BS drafted the manuscript. MZ and $\mathrm{KZ}$ provided valuable suggestions. $\mathrm{XL}$ and $J \mathrm{~L}$ discussed this manuscript. $\mathrm{HL}$ provided transcriptome data. SZ provided the research platform. JW managed and designed this research. All authors read and approved the final manuscript.

\section{Funding}

This research was funded by the National Natural Science Foundation of China (31820103012 and 31672111), the Earmarked Fund for Jiangsu Agricultural Industry Technology System (JATS [2018]277), the Earmarked Fund for China Agriculture Research System (CARS-28).

\section{Availability of data and materials}

The pear (Pyrus bretschneideri) genome sequence and RNA-seq data was downloaded from the pear genome project (http://peargenome.njau.edu.cn/).

Ethics approval and consent to participate Not applicable. 


\section{Consent for publication}

Not applicable.

\section{Competing interests}

The authors declare that they have no competing interests.

\section{Author details}

${ }^{1}$ Center of Pear Engineering Technology Research, State Key Laboratory of Crop Genetics and Germplasm Enhancement, Nanjing Agricultural University, Nanjing 210095, China. ${ }^{2}$ Shenzhen Branch, Guangdong Laboratory for Lingnan Modern Agriculture, Genome Analysis Laboratory of the Ministry of Agriculture, Agricultural Genomics Institute at Shenzhen, Chinese Academy of Agricultural Sciences, Shenzhen, China.

Received: 24 February 2020 Accepted: 17 August 2020

Published online: 21 September 2020

\section{References}

1. Naito T, Yamashino T, Kiba T, Koizumi N, Kojima M, Sakakibara H, Mizuno T. A link between cytokinin and ASL9 (ASYMMETRIC LEAVES 2 LIKE 9) that belongs to the AS2/LOB (LATERAL ORGAN BOUNDARIES) family genes in Arabidopsis thaliana. Biosci Biotechnol Biochem. 2007:71(5):1269-78

2. Rubin G, Tohge T, Matsuda F, Saito K, Scheible WR. Members of the LBD family of transcription factors repress anthocyanin synthesis and affect additional nitrogen responses in Arabidopsis. Plant Cell. 2009;21(11):356784

3. Okushima Y, Fukaki H, Onoda M, Theologis A, Tasaka M. ARF7 and ARF19 regulate lateral root formation via direct activation of $L B D / A S L$ genes in Arabidopsis. Plant Cell. 2007;19(1):118-30.

4. Han WL, Kim MJ, Nan YK, Lee SH, Kim J. LBD18 acts as a transcriptional activator that directly binds to the EXPANSIN14 promoter in promoting lateral root emergence of Arabidopsis. Plant J. 2012;73(2):212-24.

5. Shuai. The Lateral Organ Boundaries Gene Defines a Novel, Plant-Specific Gene Family. Plant physiol. 2002;129(2):747-61.

6. Matsumura Y, Iwakawa H, Machida Y, Machida C. Characterization of genes in the ASYMMETRIC LEAVES2/LATERAL ORGAN BOUNDARIES (AS2/LOB) family in Arabidopsis thaliana, and functional and molecular comparisons between AS2 and other family members. PLANT PHYSIOLOGY. 2009;151(3): 1377-89.

7. Woo LH, Young KN, Ju LD, Jungmook K. LBD18/ASL20 regulates lateral root formation in combination with LBD16/ASL18 downstream of ARF7 and ARF19 in Arabidopsis. Plant Physiol. 2009;151(3):1377-89.

8. Iwakawa H, Ueno Y, Semiarti E, Onouchi H, Kojima S, Tsukaya H, Hasebe M, Soma T, Ikezaki M, Machida C. The ASYMMETRIC LEAVES2 gene of Arabidopsis thaliana, required for formation of a symmetric flat leaf lamina, encodes a member of a novel family of proteins characterized by cysteine repeats and a leucine zipper. Plant Cell Physiol. 2002;43(5):467.

9. Mayumi I, Hiro T, Hidekazu I, Ayami N, Takaaki I, Hirokazu T, Yoko M, Irena P, Yuval E, Simon VP. Dual regulation of ETTIN (ARF3) gene expression by AS1AS2, which maintains the DNA methylation level, is involved in stabilization of leaf adaxial-abaxial partitioning in Arabidopsis. Development. 2013;140(9): 1958-69.

10. Bortiri E, Chuck G, Vollbrecht E, Rocheford T, Martienssen R, Hake $S$. Ramosa2 encodes a LATERAL ORGAN BOUNDARY domain protein that determines the fate of stem cells in branch meristems of maize. Plant Cell. 2006;18(3):574-85

11. Wang $X$, Zhang $S$, Su L, Liu X, Hao Y. A genome-wide analysis of the LBD (LATERAL ORGAN BOUNDARIES domain) gene family in Malus domestica with a functional characterization of MdLBD11. PLOS One. 2013:8(2):e57044.

12. Yuemin Z, Shizhong Z, Chengchao Z. Genomewide analysis of LATERAL ORGAN BOUNDARIES domain gene family in Zea mays. J Genet. 2014;93(1): 79-91.

13. Cao H, Liu CY, Liu CX, Zhao YL, Rui-Rui XU. Genomewide analysis of the lateral organ boundaries domain gene family in Vitis vinifera. J Genet. 2016; 95(3):1-12.

14. Wang XF, Liu X, Ling SU, Sun YJ, Zhang SZ, Hao YJ, You CX. Identification, Evolution and Expression Analysis of the LBD Gene Family in Tomato. Scientia Agricultura Sinica.2013;46(12): 2501-2513.

15. El-Gebali S, Mistry J, Bateman A, Eddy SR, Luciani A, Potter SC, Qureshi M, Richardson LJ, Salazar GA, Smart A. The Pfam protein families database in 2019. Nucleic Acids Res.2018;47(D1):D427-32.
16. Timothy Bailey CE. Fitting a mixture model by expectation maximization to discover motifs in biopolymers; 1994. p. 1994.

17. Zhao J, Zhai Z, Li Y, Geng S, Song G, Guan J, Jia M, Wang F, Sun G, Feng N, et al. Genome-Wide Identification and Expression Profiling of the TCP Family Genes in Spike and Grain Development of Wheat (Triticum aestivum L.). Frontiers in plant science. 2018:9:1282.

18. Magali L, Patrice D, Gert T, Kathleen M, Yves M, Yves VDP, Pierre R, Stephane R. PlantCARE, a database of plant cis-acting regulatory elements and a portal to tools for in silico analysis of promoter sequences. Nucleic Acids Res. 2002;30(1):325-7.

19. Fawcett JA, Steven M, Yves VDP. Plants with double genomes might have had a better chance to survive the cretaceous-tertiary extinction event. Proc Natl Acad Sci U S A. 2009;106(14):5737-42.

20. Wu J, Wang Z, Shi Z, Zhang S, Ming R, Zhu S, Khan MA, Tao S, Korban SS, Wang $\mathrm{H}$. The genome of the pear (Pyrus bretschneideri Rehd.). Genome Res. 2013;23(2):396-408.

21. Fay JC, Wyckoff $\mathrm{GJ}, \mathrm{Wu} \mathrm{Cl}$. Positive and negative selection on the human genome. Genetics. 2001;158(3):1227-34.

22. Starr TK, And SCJ, Hogquist KA. Positive and negative selection of $\mathrm{t}$ cells. Annu Rev Immunol. 2003;21(1):139-76.

23. Li X, Cheng X, Li J, Xin Q, Li L, Li'ang Y, Huang Y, Wu J. Genome-wide identification, evolution and functional divergence of MYB transcription factors in Chinese white pear ( Pyrus bretschneideri ). Plant Cell Physiol. 2016;4:4.

24. Qiao X, Li M, Li L, Yin H, Wu J, Zhang S. Genome-wide identification and comparative analysis of the heat shock transcription factor family in Chinese white pear (Pyrus bretschneideri) and five other Rosaceae species. BMC Plant Biol. 2015;15(1):12.

25. Li Q, Qiao X, Yin H, Zhou Y, Dong H, Qi K, Li L, Zhang S. Unbiased subgenome evolution following a recent whole-genome duplication in pear (Pyrus bretschneideri Rehd.). Horticulture Res. 2019;6(1):34.

26. Zhang J, Tang W, Huang Y, Niu X, Zhao Y, Han Y, Liu Y. Down-regulation of a LBD-like gene, OsIG1, leads to occurrence of unusual double ovules and developmental abnormalities of various floral organs and megagametophyte in rice. Journal of experimental botany. 2014;66(1):99112.

27. Li HH, Liu X, An J-P, Hao Y-J, Wang X-F, You C-X. Cloning and elucidation of the functional role of apple MdLBD13 in anthocyanin biosynthesis and nitrate assimilation. Plant Cell, Tissue and Organ Culture (PCTOC). 2017; 130(1):47-59.

28. Liu H, Su J, Zhu Y, Yao G, Allan AC, Ampomah-Dwamena C, Shu Q, LinWang K, Zhang S, Wu J. The involvement of PybZIPa in light-induced anthocyanin accumulation via the activation of PyUFGT through binding to tandem G-boxes in its promoter. Horticulture Res. 2019;6(1):134.

29. Cannon SB, Mitra A, Baumgarten A, Young ND, May G. The roles of segmental and tandem gene duplication in the evolution of large gene families in Arabidopsis thaliana. BMC Plant Biol. 2004:4(1):10.

30. Maher C, Stein L, Ware D. Evolution of Arabidopsis microRNA families through duplication events. Genome Res. 2006;16(4):510-9.

31. Jiang WK, Gao LZ. Prevalent role of gene features in determining evolutionary fates of whole-genome duplication duplicated genes in flowering plants. Plant Physiol. 2013;161(4):1844-61.

32. Li $X$, Tao $S$, Wei $S$, Ming $M$, Huang $X$, Zhang $S$, Wu J. The mining and evolutionary investigation of AP2/ERF genes in pear (Pyrus). BMC plant biology. 2018;18(1):46.

33. Freeling M. Bias in plant gene content following different sorts of duplication: tandem, whole-genome, segmental, or by transposition. Annu Rev Plant Biol. 2009;60(60):433-53.

34. Wu J, Wang Z, Khan MA, Shu Z, Korban SS, Ming R, Zhang S. The Genome of Pear (Pyrus bretschneideri Rehd.). In: International Plant \& Animal Genome Conference XXI; 2012. p. 2012.

35. Wang R, Ming M, Li J, Shi D, Qiao X, Li L, Zhang S, Wu J. Genome-wide identification of the MADS-box transcription factor family in pear (Pyrus bretschneideri) reveals evolution and functional divergence. PeerJ.2017;5(9): e3776.

36. Lü H, Li J, Huang Y, Zhang M, Zhang S, Wu J.Genome-wide identification, expression and functional analysis of the phosphofructokinase gene family in Chinese white pear (Pyrus bretschneideri). Gene. 2019;702:133-142.

37. Antonio CJ, John F, Mes JJ, Nayelli MM, Andy P, Angenent GC. ASYMMETRIC LEAVES2-LIKE1 gene, a member of the AS2/LOB family, controls proximaldistal patterning in Arabidopsis petals. Plant Mol Biol. 2005;57(4):559-75. 
38. Huazhao Y, Mizhen Z, Weimin W, Hongmei Y, Yaming Q, Zhuangwei W, Xicheng W. Genome-wide identification and expression analysis of auxinrelated gene families in grape. Yi chuan = Hereditas. 2015;37(7):720-30.

39. L-j B, Shan W, Kuang J-F, Feng B-H, Xiao Y-Y, Lu W-J, Chen J-Y. The Banana MaLBD (LATERAL ORGAN BOUNDARIES DOMAIN) transcription factors regulate EXPANSIN expression and are involved in fruit ripening. Plant Mol Biol Report. 2014;32(6):1103-13.

40. Sun-Hyung, Lim, Ji-Hye Song, Da-Hye Kim, Jae, Kwang, Kim Jong-Yeol. Activation of anthocyanin biosynthesis by expression of the radish R2R3MYB transcription factor gene RsMYB1.Plant cell reports. 2016;35(3):641-653.

41. Naing AH, Kim CK. Roles of R2R3-MYB transcription factors in transcriptional regulation of anthocyanin biosynthesis in horticultural plants. Plant molecular biology 2018;98(1):1-18.

42. Fan L, Xu L, Wang Y, Tang M, Liu L. Genome- and Transcriptome-Wide Characterization of bZIP Gene Family Identifies Potential Members Involved in Abiotic Stress Response and Anthocyanin Biosynthesis in Radish (Raphanus sativus L.). Int J Mol Sci. 2019;20(24):6334.

43. Voorrips RE. MapChart: software for the graphical presentation of linkage maps and QTLs. J Hered. 2002;93(1):77-8.

44. Panu A, Manohar J, Konstantin A, Delphine B, Gabor C, Edouard DC, Séverine D, Volker F, Arnaud F, Elisabeth G. ExPASy: SIB bioinformatics resource portal. Nucleic Acids Res. 2012;40(Web Server issue):W597.

45. Chen C, Xia R, Chen H, He Y. TBtools: An Integrative Toolkit Developed for Interactive Analyses of Big Biological Data. Molecular plant.2020;13(8):11941202.

46. Timothy B, Elkan C. Fitting a mixture model by expectation maximization to discover motifs in bipolymers; 1993.

47. Thompson JD, Gibson TJ, Higgins DG. Multiple Sequence Alignment Using ClustalW and ClustalX. Current Protocols in Bioinformatics.2003; 00(1):2.3.1-2. 3.22 .

48. Nicholas K, Nicholas H. GeneDoc: a tool for editing and annotating multiple sequence alignments; 1997.

49. Cummings MP. MEGA (molecular evolutionary genetics analysis); 2004

50. Huangkai Z, Shenghan G, Lercher MJ, Songnian H, Wei-Hua C. EvolView, an online tool for visualizing, annotating and managing phylogenetic trees. Nucleic Acids Res. 2012;40(Web Server issue):569-72.

51. Quinlan AR, Hall IM. BEDTools: a flexible suite of utilities for comparing genomic features. Bioinformatics. 2010;26(6):841-42.

52. Lobo I. Basic local alignment search tool (BLAST). J Mol Biol. 2012;215(3): 403-10.

53. Wang Y, Tang H, Debarry JD, Tan X, Li J, Wang X, Lee T, Jin H, Marler B, Guo H. MCScanX: a toolkit for detection and evolutionary analysis of gene synteny and collinearity. Nucleic Acids Res. 2012;40(7):e49.

54. Qiao X, Li Q, Yin H, Qi K, Li L, Wang R, Zhang S, Paterson AH. Gene duplication and evolution in recurring polyploidization-diploidization cycles in plants. Genome biology. 2019;20(1):38.

55. Zhang Z, Xiao J, Wu J, Zhang H, Liu G, Wang X, Lin D, Bbrc J. ParaAT: a parallel tool for constructing multiple protein-coding DNA alignments. Biochem Biophys Res Commun. 2012;419(4):779-81.

56. Wang D, Zhang Y, Zhang Z, Zhu J. Kaks_Calculator 2.0: a toolkit incorporating gamma-series methods and sliding window strategies. Genomics Proteomics Bioinformatics. 2010;8(1):77-80.

57. Li Q, Qiao X, Yin H, Zhou Y, Zhang S: Unbiased subgenome evolution following a recent whole-genome duplication in pear (Pyrus bretschneideri Rehd.). Horticulture Research.2019;6(1):34.

58. Huang $C$, Bo $Y$, Teng $Y$, Su J, Shu Q, Cheng Z, Zeng L. Effects of fruit bagging on coloring and related physiology, and qualities of red Chinese sand pears during fruit maturation. Sci Hortic. 2009;121(2):0-158.

59. Livak K, Schmittgen T. Analysis of Relative Gene Expression Data Using RealTime Quantitative PCR and the 2- $\Delta \Delta$ Ct Method. Methods. 2000;25(4):402408.

60. Cheng, Xue, Jia-Long, Yao, Yong-Song, Guan-Qing, Su, Liang, Wang, Li-Kun. PbrMYB169 positively regulates lignification of stone cells in pear fruit. Journal of experimental botany. 2019;70(6):1801-1814.

\section{Publisher's Note}

Springer Nature remains neutral with regard to jurisdictional claims in published maps and institutional affiliations.

\section{Ready to submit your research? Choose BMC and benefit from:}

- fast, convenient online submission

- thorough peer review by experienced researchers in your field

- rapid publication on acceptance

- support for research data, including large and complex data types

- gold Open Access which fosters wider collaboration and increased citations

- maximum visibility for your research: over $100 \mathrm{M}$ website views per year

At $\mathrm{BMC}$, research is always in progress.

Learn more biomedcentral.com/submissions 